\title{
The role of microRNAs in nasopharyngeal carcinoma
}

\author{
Gongjun Tan $•$ Xiaowei Tang $\cdot$ Faqing Tang
}

Received: 3 October 2014 / Accepted: 12 November 2014 / Published online: 27 November 2014

(C) The Author(s) 2014. This article is published with open access at Springerlink.com

\begin{abstract}
Nasopharyngeal carcinoma (NPC), a distinct type of head and neck cancer, is prevalent in Southeast Asia and southern China. Ethnic background and environmental factors contribute to the development of NPC, further complicating its pathogenesis. An increasing body of evidence indicates that microRNAs (miRNAs) play an important role in the development and progression of NPC, in particular, 32 miRNAs are involved in NPC tumorigenesis, progression, and metastasis. The causal involvement of miRNAs in NPC and their possible use as biomarkers have been extensively studied with promising results, demonstrating the diagnostic and therapeutic potential of miRNAs in NPC. In this review, we summarize the role of all the known miRNAs involved in the signaling pathway implicated in NPC.
\end{abstract}

Keywords microRNA $\cdot$ Nasopharyngeal carcinoma .

Tumorigenesis $\cdot$ Metastasis $\cdot$ Biomarker $\cdot$ Therapeutic target

\section{Introduction}

Nasopharyngeal carcinoma (NPC) is endemic in southern China and Southeast Asia with an annual incidence of 1550 cases per 100,000 [1]. Both ethnic background and

Gongjun Tan and Xiaowei Tang contributed equally to this work.

G. Tan $\cdot$ F. Tang

Medical Research Center, Xiangya Hospital, Central South

University, 87 Xiangya Road, Changsha 410008, Hunan, China

G. Tan $\cdot$ F. Tang $(\bowtie)$

Department of Clinical Laboratory, Zhuhai Hospital, Jinan

University, Zhuhai 519000, Guangdong, China

e-mail: tangfaqing33@hotmail.com

X. Tang

2011 Undergraduate, Metallurgical Science and Engineering, Central South University, 923 Lushan South Road, Changsha 410083, China environmental factors contribute to the risk of NPC development. The Chinese population emigrating to Southeast Asia or North America is considered to be at high risk. In contrast, the incidence of NPC is much lower in the Chinese individuals born in North America [2, 3]. According to global cancer statistics reported by the International Agency for Research on Cancer, over 84,000 new NPC cases occur annually, among which $80 \%$ are located in Asia and $5 \%$ in Europe. NPC is characterized by poorly or undifferentiated carcinoma. It differs from non-nasopharyngeal head and neck squamous cell carcinomas in several ways including its association with the Epstein-Barr virus (EBV) and a great propensity for distant metastases [4]. Advances in radiotherapy and comprehensive chemotherapy strategies have greatly improved outcomes in the patients with primary NPC; the 5-year survival rate increases from $50 \%$ in the 1980 s to $70 \%$ in the 1990 s $[5,6]$. However, $15-58 \%$ of NPC patients experience recurrence of the disease and have to undergo re-treatment $[7,8]$.

MicroRNAs (miRNAs or miRs) are endogenous, small, non-coding single-stranded RNAs of $\sim 22$ nucleotides in length, of which negative regulators of gene expression are small non-coding RNAs that typically inhibit the translation and reduce the stability of messenger RNAs (mRNAs). Thereby, they are involved in cellular processes such as inflammation, cell-cycle regulation, stress response, differentiation, apoptosis, and migration [9]. The annotation of miRNAs genomic positions indicates that most miRNA-coding genes are located in the introns of protein-coding genes, and the introns or exons of noncoding genes [10]. miRNAs can be organized as individual genes or localized as clusters representing miRNA families, which are commonly related in their sequence and function. miRNAs are mainly transcribed by RNA polymerase II (RNA pol II) from their own promoter or the promoter of the host gene in which they reside. The RNA pol II synthesizes large miRNA precursors called primary- 
miRNAs (pri-miRNAs) [11]. The canonical miRNA biogenesis pathway consists of two main processing steps that occur in the nucleus and the cytoplasm. pri-miRNAs are first processed into the nucleus and then cleaved into a 60 $70 \mathrm{nt}$ double-helix hairpin structure (called precursor miRNA or pre-miRNA) by the RNase III Drosha and its cofactor DGCR8 [12]. Exportin 5 mediates the transfer of the pre-miRNA into the cytoplasm [13], where the second cropping process (dicing) takes place. The pre-miRNA is first processed by the RNase III Dicer, in concert with TAR RNA binding protein or protein activator of the interferoninduced protein kinase cofactors, into a $22 \mathrm{nt}$ dsRNA with two-nucleotide 3 '-overhangs called miRNA/miRNA* duplex. It is finally unwound by a cytoplasmic helicase [14]. The mature miRNA guide is generally selected according to its thermodynamic properties, while the complementary passenger strand is usually subject to degradation. It was formerly thought that the miRNA strand is preferentially degraded; however, more recent evidence suggests that it does not simply represent a non-functional byproduct of miRNA biogenesis but can be selected as the functional strand to play significant biological roles [15].

The miRNA strand with the less stable $5^{\prime}$ end (guide strand) is preferentially selected and incorporated in the RNA-induced Silencing Complex. The mature miRNA regulates gene expression at the posttranscriptional level by binding through partial complementarity to target mRNAs [generally the 3'-untranslated regions (UTR)], which results in mRNA degradation or translation inhibition [16]. Indeed, miRNAs mainly recognize complementary sequences in the $3^{\prime}$-UTRs of their target mRNAs; however, recent studies have reported that miRNAs can also bind to the 5'-UTR or the open reading frame. Surprisingly, they can also upregulate translation under conditions of growth arrest [17, 18]. Moreover, miRNAs, as well as dsRNAs, are known to bind promoter regions at the genomic level and induce gene expression by returning to the nucleus or a hexanucleotide terminal motif-mediated transfer $[19,20]$.

Genetic variants of the miRNA biogenesis pathway are associated with the risk and/or survival in various malignancies such as bladder, ovarian, prostate, and breast cancer [21-24]. miRNAs can also be secreted in the surrounding region or the biological fluids where they are protected in liposomal-like particles and act on other cell types. Indeed, increasing evidences reveal that circulating miRNAs are associated with microvesicles - small exosomes/vesicles of endocytic origin, which are released by normal healthy or damaged cell types. In the present review, we summarize miRNAs in the carcinogenesis and metastasis of NPC, their potential as therapeutic targets and diagnostic markers, and their mediating signal pathway. This provides a novel clue for further investigating NPC.

\section{miRNAs are involved in multiple stages of NPC tumorigenesis}

miRNA expression in NPC

Since the discovery of miRNAs and their involvement in chronic lymphocytic leukemia [25], the role of miRNA dysregulation in NPC pathogenesis has been studied extensively [26]. miRNAs may function as oncogenes or tumor suppressors in the malignant progression of different tumor types. Profiling analysis indicated that a large number of miRNAs are either down- or upregulated in NPC tissues. Table 1 enlists a number of miRNAs involved in NPC, as supported by validated experimental data.

miRNA functions as a tumor suppressor to regulate NPC

Generally, cancer develops sophisticated networks of multiple signaling pathways, which contribute to their ability to progress, in some cases, and to evade treatment. Gain-of-function and loss-of-function experiments, in combination with target prediction analyses, have demonstrated that miRNAs can influence multiple steps of tumorigenesis [27]. miRNAs that function as tumor suppressors are often downregulated in NPC tissues. Since these miRNAs are negative regulators of protein-coding genes, the downregulation of miRNAs is expected to cause an upregulation of their target genes and subsequent alterations of the associated cellular pathways in NPC (Fig. 1). Among the tumor suppressor microRNAs involved in NPC, the miR-9, miR-26, miR-29, miR-200 family, and the Let-7 family are the most common.

miR-9 appears to be one of the most important players in NPC biology; while it is commonly downregulated in NPC, it is known to regulate proliferation, epithelial-mesenchymal transition (EMT), invasion, metastasis, apoptosis, and angiogenesis in other cancers (Fig. 1) [28, 29]. Mechanistically, miR-9 can directly bind to the 3'-UTR of CXCR4 to downregulate its expression. CXCR4 downregulation inhibits cell growth, migration, and invasion, whereas its overexpression rescues the suppressive effect of miR-9 [30]. In addition, miR9 can modulate the expression of interferon-induced genes and major histocompatibility complex class I molecules in human cancer cells, indicating a novel role of miR-9 in linking inflammation and cancer [31]. Ectopic expression of miR-9 dramatically inhibits the proliferative, migratory, and invasive capabilities of NPC cells both in vitro and in vivo. Low plasma levels of miR-9 are significantly correlated with increased lymphatic invasion and advanced TNM stage. Furthermore, these levels are significantly higher in posttreatment samples than in pre-treatment samples [32]. miR26a functions to suppress growth in NPC; however, miR-26a is commonly downregulated in NPC specimens and cell lines; its ectopic expression dramatically suppresses cell 
Table 1 List of miRNAs involved in NPC, their validated targets, and biological functions

\begin{tabular}{|c|c|c|}
\hline miRNAs & Validated target(s) & Main biological function(s) \\
\hline \multicolumn{3}{|c|}{ Tumor suppressors } \\
\hline miR-1 & PTMA & Induces carcinoma cell apoptosis \\
\hline miRNA let-7 & c-Myc, EZH2 & Inhibits cell proliferation and induces cell apoptosis \\
\hline miR-9 & $\mathrm{CXCR} 4$ & $\begin{array}{l}\text { Regulates proliferation, EMT, invasion, metastasis, apoptosis, and tumor } \\
\text { angiogenesis }\end{array}$ \\
\hline miR-26a & $\mathrm{EZH} 2, \mathrm{c}-\mathrm{Myc}$ & Suppresses cell proliferation and colony formation \\
\hline $\operatorname{miR}-29 c$ & TIAM1 & Inhibits cell migration and invasion \\
\hline miR-98 & EZH2 & Inhibits relapse \\
\hline miR-124 & Foxq1 & Inhibits cell growth, migration, and invasion \\
\hline $\operatorname{miR}-138$ & CCND1 & Suppresses cell proliferation and colony formation \\
\hline $\operatorname{miR}-184$ & BCL2, c-Myc & Suppresses cell proliferation \\
\hline miR-200 & ZEB2, CTNNB1, Notch1 & Regulates EMT, migration, and invasion \\
\hline miR-204 & Stat-3, CDC42 & Regulates invasion \\
\hline $\operatorname{miR}-216 b$ & PKCá, K-Ras & Suppresses proliferation and invasion \\
\hline miR-375 & MTDH & Suppresses relapse \\
\hline $\operatorname{miR}-451$ & MIF & Regulates NPC cell growth and invasion \\
\hline \multicolumn{3}{|l|}{ Onco-miRNAs } \\
\hline miR-10b & MMP-9 & Promotes mobility and invasion \\
\hline miR-18a & Dicer1, c-Jun, c-Myc, & Lymph node metastasis \\
\hline $\operatorname{miR}-18 b$ & CTGF & Promotes cell growth \\
\hline miR-21 & BCL2 & Promotes migration and proliferation \\
\hline miR-30a & E-cadherin & Increases the capability of metastasis and invasion \\
\hline miR-93 & TGFâR2 & Promotes cell proliferation, invasion, and metastasis \\
\hline miR-141 & BRD3, PTEN, SPLUNC1 & Promotes cell growth, migration, and invasion \\
\hline miR-144 & PTEN & Promotes migration and invasion \\
\hline miR-149 & E-cadherin & Promotes mobility and invasion \\
\hline miR-155 & JMJD1A, BACH1 & Stimulates cell proliferation, colony formation, cell migration, and invasion \\
\hline miR-205 & PTEN & Attenuates cell apoptosis post-irradiation \\
\hline miR-214 & LTF, Bim & Promotes NPC cell proliferation, invasion, and metastasis \\
\hline $\operatorname{miR}-378$ & TOB2 & Promotes cell proliferation, colony formation, migration, and invasion \\
\hline $\operatorname{miR}-421$ & FOXO4 & Induces cell growth and apoptosis resistance \\
\hline miR-663 & $\mathrm{p} 21$ & Promotes cellular G1/S transition \\
\hline
\end{tabular}

PTMA prothymosin alpha or ProT alpha, $I F N$ interferon, $M H C$ major histocompatibility complex, $C X C R 4$ chemokine (C-X-C motif) receptor 4, CCND1 cyclin D1, ZEB E-box binding homeobox, $C T N N B 1$ catenin (cadherin-associated protein), beta 1, CDC42 cell division cycle $42, P K C$ protein kinase C, $M I F$ macrophage migration inhibitory factor, $M M P-9$ matrix metalloproteinase-9, $C T G F$ connective tissue growth factor, $T G F \beta R 2$ transforming growth factor- $\beta$ receptor II, $B R D 3$ bromodomain containing 3, PTEN phosphatase and tensin homolog, SPLUNC1 short palate, lung, and nasal epithelium clone 1, JMJD1A, BACH1 BTB and CNC homology 1, LTF lactotransferrin, TOB2 transducer of ERBB2, FOXO4 forkhead box O 4

proliferation and colony formation by inducing $\mathrm{G}_{1}$ cell-cycle arrest. In addition, miR-26a strongly reduces the expression of the EZH2 (Zeste homolog 2) oncogene in NPC cells (Fig. 1) [33]. Mechanistically, miR-26a suppresses the expression of c-Myc, cyclins D3 and E2, and the cyclin-dependent kinases (CDK), CDK4 and CDK6, enhances the expression of the CDK inhibitors p14(ARF) and p21(CIP1) in an EZH2dependent manner. The antimetastatic functions of miR-26a are primarily mediated by repressing EZH2 expression [34]. miR-29c is also downregulated in NPC; its ectopic expression inhibits NPC cell migration and invasion in vitro and suppresses the formation of lung metastases in vivo. Studies using luciferase reporter assays have confirmed that $\mathrm{T}$ cell lymphoma invasion and metastasis 1 (TIAM1) is a miR-29c target gene [35]. Reduced expression of miR-29c is positively correlated with therapeutic resistance in NPC patients. Mechanistically, miR-29c substantially enhances the sensitivity of NPC cells to ionizing radiation (IR) and cisplatin treatment through the repression of antiapoptotic factors, Mcl-1 and $\mathrm{Bcl}-2$ [36]. Moreover, miR-98 is also found to be underexpressed in relapsed NPC patient samples. Using luciferase-based assays, miR-98 has been validated as a bona 
Fig. 1 Schematic illustration of microRNAs (miRNA) involved in nasopharyngeal carcinoma (NPC). miRNAs are involved in nasopharyngeal epithelium carcinogenesis, NPC cell apoptosis, proliferation epithelial-mesenchymal transition, migration, and invasion. Some miRNAs are associated with clinical prognosis. Plus sign upregulated miRNA; minus sign downregulated miRNA

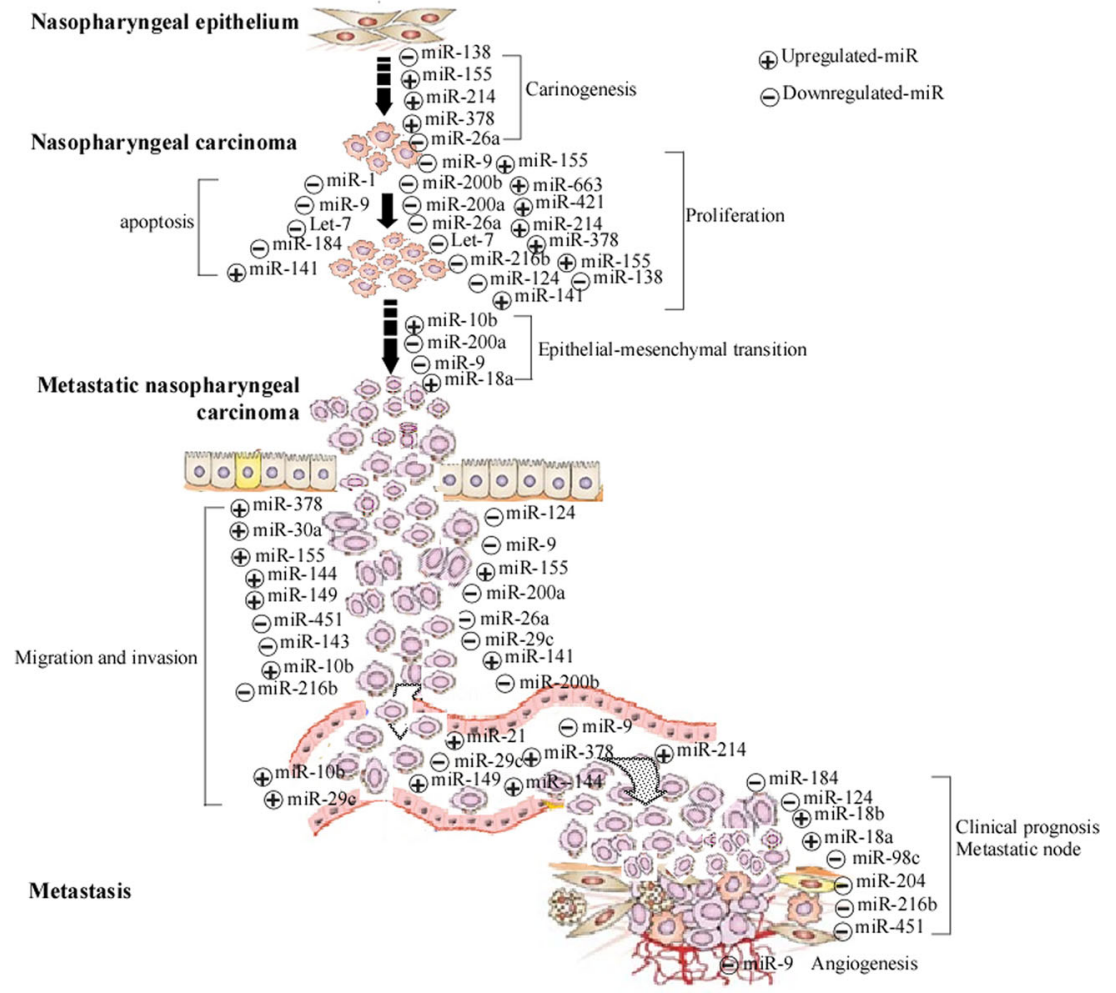

fide regulator of the expression of EZH2, which is associated with a higher risk of relapse in NPC patients [37].

miR-200 family is often downregulated in NPC. The overexpression of miR-200a inhibits the growth, migration, and invasion of C666-1 cells (Fig. 1), whereas its knock-down stimulates these processes in CNE-1 cells. It is speculated that E-box binding homeobox 2(ZEB2), catenin (cadherin-associated protein), and beta $1(\mathrm{CTNNB} 1)$ are the functional downstream targets of miR-200a. The knock-down of ZEB2 solely impedes NPC cell migration and invasion, whereas CTNNB1 suppression inhibits NPC cell growth. These suggest that the inhibitory effects of miR-200a on NPC cell growth, migration, and invasion are mediated by distinct targets and pathways [38]. miR-200a also regulates EMT as well as stem cell-like transition in NPC cells (Fig. 1). Stable knockdown of miR200a promotes the transition of epithelium-like CNE-1 cells to the mesenchymal phenotype. Similarly, stable overexpression of miR-200a transfers the mesenchyme-like C666-1 cells to the epithelial state along with a significant reduction of their stem cell-like features. Mechanistically, miR-200a controls EMT by targeting ZEB2, and it also regulates the stem celllike transition differentially and specifically through $\beta$-catenin signaling [39]. EBV-encoded EB nuclear antigen 1 (EBNA1) inhibits the expression of miR-200a and miR-200b, which results in the upregulation of target genes expression and zinc finger E-box binding homeobox 1 (ZEB1) and ZEB2 [40]. miR-200b is significantly downregulated in the NPC tissues and cell lines. Gain-of-function and loss-of-function studies have demonstrated that miR-200b suppresses NPC cell growth, migration, and invasion in vitro (Fig. 1). Importantly, the overexpression of miR-200b effectively represses tumor growth in nude mouse models. Furthermore, integrated analysis has identified Notch1 as a direct and functional target of miR-200b. Overexpression of Notch1 reverses the inhibitory effect of miR-200b on NPC cell growth and invasion [41].

The Let-7 family is one of the best known miRNA families in cancer biology. It regulates numerous oncogenes like RAS, MYC, and high-mobility group AT-hook 2 (HMGA2) [42, 43]. NPC cells exhibit reduced levels of miRNA Let-7 (Let-7a, Let-7-b, Let-7-d, Let-7-e, Let-7-g, and Let-7-i). Ectopic expression of the Let-7 family inhibits NPC cell proliferation by downregulating c-Myc expression. Demethylation treatment of NPC cells causes the activation of Let-7 expression in poorly differentiated NPC cells [44]. EZH2 is a direct target of Let-7a, and the in vitro inhibition of EZH2 by Let-7a and/or EZH2 siRNA attenuates NPC cell growth, inhibits cell proliferation, and induces cell apoptosis, respectively (Fig. 1) [45].

Besides the afore-mentioned microRNAs, miR-204, miR216b, miR-143, miR-375, miR-451, miR-375, miR-1, miR124, miR-138, and miR-184 are known to be downregulated in NPC cells and tissues (Fig. 1). Reduced expression of miR204 is strongly associated with a more aggressive and poor prognostic phenotype of NPC. EBV-encoded latent membrane protein 1 (LMP-1) suppresses miR-204 expression by activating Stat-3 and enhances cell division cycle 42 (CDC42) 
activity to facilitate the invasion of NPC [46]. Similarly, decreased miR-216b expression is directly related to advanced clinical stage and lymph node metastasis in NPC. Both in vitro and in vivo assays have revealed that miR-216b attenuates NPC cell proliferation, invasion (Fig. 1), and tumor growth in nude mice. miR-216b exerts its tumor suppressor function by inhibiting the KRAS-related (protein kinase B) AKT and extracellular signal-regulated kinase (ERK) pathways [47]. Additionally, the miR-216b-mediated downregulation of protein kinase $\mathrm{C} \alpha(\mathrm{PKC} \alpha)$ suppresses the proliferation and invasion ability of CNE2 cells; moreover, overexpressed PKC $\alpha$ can partially reverse the inhibitory effect of miR$216 \mathrm{~b}$ on cell proliferation [48]. Patients with low miR-451 expression have poor overall and disease-free survival compared with those with high miR-451 expression. Macrophage migration inhibitory factor, a direct target of miR-451, regulates NPC cell growth and invasion [49]. miR-143 plays a role in modulating the invasiveness and metastasis of NPC; its overexpression causes a significant reduction of the adhesion ability [50]. Studies using dual-luciferase reporter assays showed that miR-143 directly binds to the 3'-UTR of GLI3. In addition, results from qRT-PCR and Western blot analysis demonstrated that the expression of miR-143 is negatively correlated to GLI3 and suppresses the migration of 5-8 F cells [51]. In the NPC samples, miR-375 expression is significantly reduced, while metadherin (MTDH) is significantly increased. NPC patients with high MTDH experience significantly lower survival, as well as higher distant relapse rates. Luciferase assays have also corroborated MTDH as a target of miR-375 [52]. miR-1 can induce NPC cell apoptosis by directly targeting the prothymosin alpha gene, in which siRNA and miR-1 accelerate the apoptotic process in cells treated with apoptosis inducers [53]. miR-124 is also commonly downregulated in NPC specimens and cell lines, and its expression is inversely correlated with clinical stages. Foxq1 is a novel direct target of miR-124, and its knockdown inhibits cell growth, migration, and invasion (Fig. 1) [54]. miR-138 is another downexpressed miRNA, and its ectopic expression suppresses NPC cell proliferation and colony formation, and also inhibits tumorigenesis in vivo (Fig. 1). Cyclin D1 is a direct target of miR-138, and its mRNA levels are inversely correlated with miR-138 expression [55]. miR-184, which is modulated by programmed cell death 4 (PDCD4), directly targets BCL2 and C-MYC and participates in PDCD4mediated suppression of cell proliferation and survival in NPC [56].

Onco-miRNAs are involved in the regulation of NPC

In tumorigenesis of NPC, some miRNAs called oncomiRNAs, function as oncogenes, for example, miR-18, miR214, miR-155, and miR-141. miR-18, a member of the oncogenic miR-17-92 cluster, acts as an onco-miR in NPC development (Fig. 1). Indeed, miR-18a is upregulated in NPC samples and cell lines [57]. Clinical parameter studies showed that miR-18a levels are correlated with advanced stages of NPC, lymph node metastasis, EBV infection, and a higher death rate. miR-18a negatively regulates Dicer1 by binding to its 3'-UTR, resulting in global downregulation of miR-200 family and miR-143 expression. The EMT marker E-cadherin and oncogene K-Ras are aberrantly expressed after miR-18a transduction, and these alterations are directly induced by the downregulation of miR-200 family and miR-143 [58]. miR-18b directly suppresses the expression of connective tissue growth factor (CTGF) in NPC, and its downregulation is significantly associated with NPC progression and poor prognosis. In fresh clinical specimens, miR-18b was widely overexpressed and inversely correlated with CTGF expression [59]. miR-141 was also upregulated in the NPC specimens. Both c-Myc knockdown and the re-expression of the host defensive protein short palate, lung, and nasal epithelium clone 1 (SPLUNC1) can downregulate miR-141. The miR-141 inhibition, in turn, affects cell cycle, apoptosis, growth, migration and invasion. Studies using luciferase reporter assays have confirmed that bromodomain containing 3(BRD3), ubiquitin-associated protein 1 (UBAP1), and PTEN are the potential targets of miR-141 [60]. SPLUNC1 functions at the very early stage of NPC carcinogenesis. It regulates NPC cell proliferation, differentiation, and apoptosis through miR-141, which in turn regulates the expression of PTEN and $p 27$. This signaling axis is negatively regulated by the EBVcoded gene LMP1 [61]. Although miR-155 is upregulated in the two EBV-negative NPC cell lines, Chinese nasopharyngeal carcinoma cell line 1 and nasopharyngeal carcinoma cell line from Taiwan (TW03), EBV-encoded LMP1, and LMP2A may further enhance miR-155 expression. Jumonji Domain 1A (JMJD1A) and BTB and CNC homology 1 (BACH1), putative targets of miR-155, can be downregulated by miR155 mimics, while miR-155 inhibitor upregulates the expression of JMJD1A in the NPC cell lines. The downregulation of JMJD1A is significantly correlated with the $\mathrm{N}$ stage of the TNM classification, a lower 5-year survival rate, and a lower 5-year disease-free survival rate of NPC patients [62]. In addition, miR-155 expression is upregulated in EBV-positive NPC tissue samples and is correlated with plasma DNA copies of LMP1. The expression of miR-155 is also upregulated in the NPC cell lines when transfected with an LMP1expressing plasmid. Upregulated miR-155 stimulates NPC cell proliferation, colony formation, cell migration, and invasion [63].

miR-214 is known to be overexpressed in NPC cell lines and tissues. miR-214 not only promotes NPC cell proliferation and invasion in vitro but also accelerates tumor formation and lung metastasis (Fig. 1). The expression of miR-214 is upregulated in NPC samples, especially in metastasis-prone NPC tumor tissues, while the 
lactotransferrin (LTF) expression level is negatively correlated with that of miR-214. These suggest that miR-214 targeting is partly responsible for the LTF downregulation in the NPC specimens [64]. The silencing of miR-214 by locked-nucleic-acid-anti-miR-214 in NPC cells results in the enhancement of apoptosis and suppression of in vitro cell proliferation and in vivo tumor growth in nude mice. Luciferase reporter assays have been performed to identify Bim as a direct target of miR-214 [65].

In addition to the above miRNAs, miR-421, miR-10b, miR-21, miR-30a, miR-93, miR-144, miR-146a, miR-149, miR-378, and miR-663 are also known to play oncogenic roles in NPC tumorigenesis (Fig. 1). Overexpression of miR-421 inhibits the forkhead box protein O4 (FOXO4) signaling pathway, and it downregulates p21, p27, Bim, and ligand (FASL) expression by directly targeting the 3'UTR of FOXO4 [66]. miR-10b is highly expressed in EBV-LMP1-positive NPC cells, and its expression is downregulated by silencing LMP1 or Twist [67]. miR$10 \mathrm{~b}$ mimics can promote the mobility and invasion of NPC cell lines, while miR-10b inhibitors impede this invasion. In addition, the expression of genes related to migration and invasion, such as E-cadherin, vimentin, and matrix metalloproteinase-9 $(M M P-9)$, is different in CNE-2 cells transfected with miR-10b mimics or treated with miR-10b inhibitors. And miR-10b is found to upregulate $M M P-9$, while knockdown of miR-10b significantly downregulates E-cadherin expression [68]. miR-21 is an onco-miR, whose levels are elevated in the NPC tissues. Consequently, cell migration is notably inhibited by the downregulation of miR-21 in vitro, while miR-21 inhibitors can downregulate B cell CLL/lymphoma 2 (BCL2) expression. This suggests that BCL2 may be a target gene for the initiation and development of NPC [69]. The expression of miRNA-146a in human NPC was found to be elevated by EBV-associated antigen LMP1 probably through the activation of the miRNA-146a promoter [70]. A single-nucleotide polymorphism in miRNA-146a is associated with an increased risk of NPC [71]. miR-378 is commonly upregulated in both NPC tissues and NPC, although this is opposite to the reported results in plasma. Functional studies have shown that the upregulation of miR-378 dramatically promotes cell proliferation, colony formation, migration, and invasion in vitro, as well as tumor growth in vivo. Mechanistic investigations reveal that miR-378 overexpression can downregulate the expression of Transducer of ERBB2 (TOB2), a potential tumor suppressor, while miR-378 silencing can enhance the TOB2 expression [72]. miR-663 is found to be upregulated in NPC cells. Inhibition of miR-663 impairs the in vitro proliferation of NPC cells and the in vivo tumor growth of xenografts in nude mice. Mechanistically, miR-663 directly targets p21 (WAF1/CIP1) to promote cellular G1/S transition, since the inhibitory effects of miR-663 on the G1/S transition can be rescued by 21 (WAF1/CIP1) silencing [73].

\section{Metastatic miRNAs in NPC}

To successfully metastasize, a tumor cell must accomplish a complex set of processes including invasion, survival, and arrest in the circulatory system, as well as colonization of foreign organs. Despite great advances in the knowledge of metastasis biology, the molecular mechanisms are not completely understood. Remarkably, a number of miRNAs exhibit a regulatory role in the metastatic program. miRNAs can promote or suppress various steps in the migration and metastasis of cancer cells [74], thereby affecting key steps such as EMT, migration, and angiogenesis (Fig. 1).

NPC exhibits invasive and metastatic features, and approximately $90 \%$ of NPC patients show cervical lymph node metastasis at the time of initial diagnosis [75]. miR-93 directly targets transforming growth factor- $\beta$ receptor II, thereby promoting NPC cell proliferation, invasion, and metastasis [76]. miR-378 dramatically promotes cell colony formation, migration, and invasion in vitro. miR-378 overexpression and silencing can downregulate or enhance the expression of the tumor suppressor TOB2, respectively [72]. miR-30a increases the capability of metastasis and invasion of the NPC tumor cells, both in vivo and in vitro, by targeting E-cadherin [77]. miR-155 stimulates NPC cell proliferation, colony formation, cell migration, and invasion by downregulating the expression of JMJD1 A and BACH1 [63]. miR-144 is inversely correlated with the expression of tumor suppressor gene phosphatase and tensin homolog (PTEN) in the NPC specimens and cell lines; consequently, miR-144 suppresses the expression of PTEN to increase the expression of phosphorylated protein kinase $\mathrm{B}$ (pAkt) and cyclin D1, and promote G(1)-phase transition and then decreases the expression of E-cadherin to promote migration and invasion [78]. The expression of miR-149 is higher in NPC cell lines 5-8 F with high metastasis ability, and it promotes their mobility and invasion by downregulating the expression of E-cadherin [79].

Conversely, a few miRNAs serve as metastasis inhibitors; miR-200a inhibits the growth, migration, and invasion of C666-1 cells and conversely regulates EMT [39]. miR-9 is commonly downregulated in NPC [31]. Ectopic expression of miR-9 dramatically inhibits the proliferative, migratory, and invasive capacities of NPC cells in vitro and in vivo. The low plasma level of miR-9 is significantly correlated with increased lymphatic invasion and advanced TNM stage [32]. miR-9 also inhibits cell growth, migration, and invasion by regulating chemokine (C-X-C motif) receptor 4(CXCR4) expression [30]. miR-29c inhibits NPC cell migration and 
invasion in vitro and suppresses lung metastases in vivo by targeting TIAM1 [35].

\section{EBV-encoded miRNA in NPC}

The herpes virus EBV is implicated in NPC and other human malignancies. EBV-encoded miRNAs are the first group of viral miRNAs to be identified. Although the close association of NPC with EBV infection has been known for more than four decades, the exact role of EBV in the pathogenesis of NPC malignancy remains unclear. Utilizing the deep sequencing technology to characterize the EBV miRNA transcriptome in clinical NPC tissues, more than 110,000 sequence reads and 44 Epstein-Barr virus encoded microRNA (EBV BART) were identified. The miRNA sequence analysis revealed that most of the highly abundant EBV miRNAs share their seed sequences (2-7 nt) with human miRNAs; it suggests that the seed sequence content may be an important factor underlying the differential accumulation of BART miRNAs [80]. NPC tumors are known to express a number of EBV-encoded proteins; they also express a large number of virus-encoded miRNAs, the most abundant of which are encoded from the BamHI-A region of the viral genome; they are thus called as BART miRNAs [81]. EBV-miR-BART1 is involved in regulating the metabolism-associated genes in NPC [82]. miRBART17 is significantly more abundant in the plasma samples from NPC patients compared with those from non-NPC subjects [83]. miR-BART3 is abundantly expressed in NPC cells. The target site of miR-BART3 is located in the 3'-UTR of the determination of interleukin 4 commitment 1 (DICE1) tumor suppressor transcript, which is usually underexpressed in EBV-expressing NPC tissues [84]. The plasma levels of EBV-miR-BART7 are significantly higher in NPC patients compared with those from healthy individuals. The expression of EBV-miR-BART7 enhances the proliferation, migration, and invasion of NPC cells in vitro. Furthermore, NPC cells expressing EBV-miR-BART7 are more resistant to cisplatin [85]. miR-BART22 is highly expressed in the NPC tissues, and it may inhibit MAP3K5 expression, thereby reducing the phosphorylation of mitogen-activated protein kinase pathway downstream molecular and then inhibit NPC cell apoptosis and differentiation, and finally prevent the NPC cells from immune surveillance [86]. miR-BART9 is highly expressed in the NPC tissues and EBV-positive NPC cells but not in the normal tissues. Notably, the level of miR-BART9 expression was higher than that of miR-21 in eight of the nine NPC tissues examined.

miRNAs as therapeutic targets or tools

Besides influencing practically all the biological processes including proliferation and apoptosis, miRNAs have great potential as therapeutic agents. Possibly, the most important advantage of miRNAs in comparison with conventional approaches that target single genes is their ability to target multiple molecules, frequently in the context of a network. This renders them extremely efficient in regulating distinct biological processes relevant to normal and malignant cell biology.

By miRNA plasmid constructed artificially, miRNA can effectively interfere with NPC cells by downregulating the expression of VEGF thus inhibiting the growth of tumor xenografts in vivo. Future application of miRNA in the gene therapy of NPC might be expected [87]. Forced expression of miR-125a-5p is known to enhance proliferation, migration, and invasion of HONE1 cells. NPC patients exhibit significantly higher expression level of miR-125a-5p than healthy controls. miR-125a-5p and curcumin are known to inhibit or upregulate the expression of tumor protein 53 (TP53), respectively. Taken together, these results indicate that curcumin exerts inhibitory effects on NPC by inhibiting the expression of miR-125a-5p and subsequently enhancing the expression of TP53 [88].

Low expression of miR-29c is positively correlated with therapeutic resistance in NPC patients; consequently, miR-29c substantially enhances the sensitivity of NPC cells to IR and cisplatin treatment by promoting apoptosis. It also represses the expression of anti-apoptotic factors, $\mathrm{Mcl}-1$ and $\mathrm{Bcl}-2$, in the NPC tissues and cell lines [36].

Gefitinib is found to inhibit the proliferation of two NPC cell lines in vitro and in vivo, wherein HK-1 cells are more sensitive to gefitinib than human nasopharyngeal carcinoma cell line 1 (HNE-1) cells. In addition, gefitinib treatment elevates the miR-125a-5p expression in the two cell lines and the serum of NPC tumor-bearing mice. This phenomenon is weak in the HNE-1 cells and strong in the HK-1 cells. Similarly, miR-125a-5p overexpression improves the antiproliferative and pro-apoptotic effects of gefitinib on the NPC cells, while its downregulation abrogates these effects. MiR-125a-5p is also known to increase p53 protein expression in HNE-1 cells and decrease Her2 protein expression in HNE-1 and HK-1 cells [89].

Interestingly, miRNAs are known to influence cellular responses to specific therapy including radiotherapy. Radiosensitivity and radiation dose of X-ray can significantly affect the expression of miR-7 in the NPC cells, indicating that miR-7 plays an important role in the radioresistance of NPC cells. Thus, suppressed miR-7 expression may elevate the radiosensitivity of NPC cells [90]. miR-205, which is elevated in the radioresistant NPC cell line CNE-2R, is known to regulate the expression of PTEN, a tumor suppressor. Introducing miR-205 into CNE-2 cells suppresses PTEN protein expression following the activation of AKT; it increases foci formation and reduces post-irradiation apoptosis. On the other hand, knocking down miR-205 in CNE-2R cells compromises the inhibition of PTEN and increases cell apoptosis 
[91]. miRNA-324-3p contributes to the radioresistance of NPC by regulating the wingless-type MMTV integration site family, member 2B (WNT2B) signaling pathway. Thus, both miRNA-324-3p and WNT2B are potential biomarkers for NPC radioresistance [92].

miRNAs as diagnostic tools

The need for clinical biomarkers for early diagnosis of NPC is extremely urgent; it should be considered that the survival and prognosis of NPC patients depends on the stages of the tumor at the time of detection. Some miRNAs that play an important role or highly express in NPC tumorigenesis may also serve as diagnostic or prognostic biomarkers of NPC. For example, most NPC patients with a poor outcome exhibit a high expression (>median) of miR-548q (70.6\%) and miR-483-5p $(64.7 \%)$ in their tissue samples, suggesting that miR-548q and miR-483-5p are potential biomarkers of NPC. Combining the receiver operating characteristic (ROC) analyses of these two miRNAs, an area under the ROC curve of 0.737 with $67.1 \%$ sensitivity and $68.0 \%$ specificity was obtained, which demonstrates the preliminary diagnostic value of plasma miRNAs [93]. The low level of plasma miR-9 is significantly correlated with increased lymphatic invasion and advanced TNM stage. Thus, plasma miR-9 could distinguish the locoregional from the metastatic NPC cases with high sensitivity and specificity. Furthermore, plasma levels of miR-9 are significantly elevated in post-treatment samples compared with pre-treatment samples. Thus, plasma miR-9 may serve as a useful biomarker to predict NPC metastasis and to monitor tumor dynamics [32]. NPC patients with low miR-483-5p and miR-103 expression have a better 5-year prognosis than those with a high expression; similarly, patients with a low concentration of miR-29a and let-7c have poorer prognosis [94]. Dicer and Drosha mRNA are significantly downregulated in NPC tissue specimens and cell lines when compared with controls, and the low expression of the Dicer and Drosha proteins is significantly correlated with shorter progressionfree survival and overall survival (OS) of NPC patients [95].

Four serum miRNAs including miR-22, miR-572, miR638 , and miR-1234 were found to be differentially altered in NPC, and they were used to construct a miRNA signature. The combination of this miRNA signature and TNM stage was found to have a better prognostic value than the TNM stage or miRNA signature alone [96]. The miRNAs were found to be expressed differentially in the serum of NPC patients compared with the healthy controls. Based on these, a diagnosis equation with $\mathrm{Ct}$ difference method has been established to distinguish NPC cases from non-cancerous controls and validated for high sensitivity and specificity [97]. A signature of five miRNAs, each significantly associated with disease-free survival (DFS), was identified in the training set. A risk score from the signature and patients classified as high risk or low risk is calculated. Compared with the patients with low-risk scores, the patients with high risk scores in the training set have shorter DFS, distant metastasis-free survival, and OS [98]. Research from the Wang group indicated differential miRNA expression during tumor relapse, demonstrating the potential use of miRNAs in the classification of the repeated recurrence of NPC, beyond the histological approach [99].

\section{Conclusions}

In summary, it is evident that miRNAs not only represent an additional level of complexity in the molecular portrait of NPC, contributing to tumor comprehension and subclassification, but also represent easily detectable biomarkers to predict prognosis and response to therapy. It is essential to validate their potential as biomarkers in different cohorts of samples, taking into account the evidence that profiling signatures are probably more statistically significant that single miRNAs in predicting outcomes. Moreover, even though the available, up-to-date data are almost exclusively from pre-clinical samples, miRNAs are promising therapeutic and diagnostic tools in NPC research.

Acknowledgments The authors acknowledge financial support from the National Natural Science Foundation of China (81071718, 81402368, 81402265, and 81372282), Fundamental Research Funds for the Guangdong Province (2011B061300053, S2013010013360), Zhuhai Best Medical Instrument Appliance Inc., and the Foundation of State Key Laboratory of Oncology in South China (HN2011-04).

Author contributions Gongjun Tan and Xiaowei Tang contributed to analysis and interpretation of data and drafting of the manuscript. Faqing Tang contributed to study concept and design, analysis and drafting of the manuscript.

\section{Conflicts of interest None}

Open Access This article is distributed under the terms of the Creative Commons Attribution License which permits any use, distribution, and reproduction in any medium, provided the original author(s) and the source are credited.

\section{References}

1. Zhang L, Chen QY, Liu H, Tang LQ, Mai HQ. Emerging treatment options for nasopharyngeal carcinoma. Drug Des Dev Ther. 2013;7: $37-52$.

2. Buell P. The effect of migration on the risk of nasopharyngeal cancer among chinese. Cancer Res. 1974;34:1189-91.

3. Wei WI, Sham JS. Nasopharyngeal carcinoma. Lancet. 2005;365: 2041-54.

4. Lee AW, Ng WT, Chan YH, Sze H, Chan C, Lam TH. The battle against nasopharyngeal cancer. Radiother Oncol. 2012;104:272-8. 
5. Su SF, Han F, Zhao C, Huang Y, Chen CY, Xiao WW, et al. Treatment outcomes for different subgroups of nasopharyngeal carcinoma patients treated with intensity-modulated radiation therapy. Chin J Cancer. 2011;30:565-73.

6. Lee AW, Foo W, Law SC, Peters LJ, Poon YF, Chappell R, et al. Total biological effect on late reactive tissues following reirradiation for recurrent nasopharyngeal carcinoma. Int J Radiat Oncol Biol Phys. 2000;46:865-72.

7. Yang TS, Ng KT, Wang HM, Wang CH, Liaw CC, Lai GM. Prognostic factors of locoregionally recurrent nasopharyngeal carcinoma-a retrospective review of 182 cases. Am J Clin Oncol. 1996;19:337-43.

8. Chang JT, See LC, Liao CT, Ng SH, Wang CH, Chen IH, et al. Locally recurrent nasopharyngeal carcinoma. Radiother Oncol. 2000;54:135-42.

9. Di Leva G, Garofalo M, Croce CM. MicroRNAs in cancer. Annu Rev Pathol. 2014;9:287-314.

10. Lin SL, Kim H, Ying SY. Intron-mediated RNA interference and microRNA (miRNA). Front Biosci. 2008;13:2216-30.

11. Lee Y, Kim M, Han J, Yeom KH, Lee S, Baek SH, et al. MicroRNA genes are transcribed by RNA polymerase II. EMBO J. 2004;23: 4051-60.

12. Lee Y, Ahn C, Han J, Choi H, Kim J, Yim J, et al. The nuclear RNase III Drosha initiates microRNA processing. Nature. 2003;425:415-9.

13. Yi R, Qin Y, Macara IG, Cullen BR. Exportin-5 mediates the nuclear export of pre-microRNAs and short hairpin RNAs. Genes Dev. 2003;17:3011-6.

14. Hammond SM. Dicing and slicing: the core machinery of the RNA interference pathway. FEBS Lett. 2005;579:5822-9.

15. Bhayani MK, Calin GA, Lai SY. Functional relevance of miRNA sequences in human disease. Mutat Res. 2012;731: 14-9.

16. Vasudevan S, Tong Y, Steitz JA. Switching from repression to activation: microRNAs can up-regulate translation. Science. 2007;318: 1931-4.

17. Moretti F, Thermann R, Hentze MW. Mechanism of translational regulation by mir- 2 from sites in the $5^{\prime}$ untranslated region or the open reading frame. RNA. 2010;16:2493-502.

18. Orom UA, Nielsen FC, Lund AH. Microrna-10a binds the 5'utr of ribosomal protein mRNAs and enhances their translation. Mol Cell. 2008;30:460-71.

19. Place RF, Li LC, Pookot D, Noonan EJ, Dahiya R. Microrna-373 induces expression of genes with complementary promoter sequences. Proc Natl Acad Sci U S A. 2008;105:1608-13.

20. Hwang HW, Wentzel EA, Mendell JT. A hexanucleotide element directs microRNA nuclear import. Science. 2007;315:97-100.

21. Yang H, Dinney CP, Ye Y, Zhu Y, Grossman HB, Wu X. Evaluation of genetic variants in microRNA-related genes and risk of bladder cancer. Cancer Res. 2008;68:2530-7.

22. Liang D, Meyer L, Chang DW, Lin J, Pu X, Ye Y, et al. Genetic variants in microRNA biosynthesis pathways and binding sites modify ovarian cancer risk, survival, and treatment response. Cancer Res. 2010;70:9765-76

23. Liu J, Liu J, Wei M, He Y, Liao B, Liao G, et al. Genetic variants in the microRNA machinery gene gemin 4 are associated with risk of prostate cancer: a case-control study of the Chinese Han population. DNA Cell Biol. 2012;31:1296-302.

24. Sung H, Jeon S, Lee KM, Han S, Song M, Choi JY, et al. Common genetic polymorphisms of microRNA biogenesis pathway genes and breast cancer survival. BMC Cancer. 2012;12: $1471-2407$.

25. Calin GA, Sevignani C, Dumitru CD, Hyslop T, Noch E, Yendamuri $\mathrm{S}$, et al. Human microRNA genes are frequently located at fragile sites and genomic regions involved in cancers. Proc Natl Acad Sci U S A. 2004;101:2999-3004.
26. Chen HC, Chen GH, Chen YH, Liao WL, Liu CY, Chang KP, et al. MicroRNA deregulation and pathway alterations in nasopharyngeal carcinoma. Br J Cancer. 2009;100:1002-11.

27. Iorio MV, Croce CM. MicroRNA involvement in human cancer. Carcinogenesis. 2012;33:1126-33.

28. Luo H, Zhang H, Zhang Z, Zhang X, Ning B, Guo J, et al. Downregulated mir-9 and mir-433 in human gastric carcinoma. J Exp Clin Cancer Res. 2009;28:1756-9966.

29. Ma L, Young J, Prabhala H, Pan E, Mestdagh P, Muth D, et al. Mir-9, a myc/mycn-activated microRNA, regulates E-cadherin and cancer metastasis. Nat Cell Biol. 2010;12:247-56.

30. Lu J, Luo H, Liu X, Peng Y, Zhang B, Wang L, et al. Mir-9 targets cxcr4 and functions as a potential tumor suppressor in nasopharyngeal carcinoma. Carcinogenesis. 2014;35:554-63.

31. Gao F, Zhao ZL, Zhao WT, Fan QR, Wang SC, Li J, et al. Mir-9 modulates the expression of interferon-regulated genes and MHC class I molecules in human nasopharyngeal carcinoma cells. Biochem Biophys Res Commun. 2013;431:610-6.

32. Lu J, Xu X, Liu X, Peng Y, Zhang B, Wang L, et al. Predictive value of mir-9 as a potential biomarker for nasopharyngeal carcinoma metastasis. Br J Cancer. 2014;110:392-8.

33. Lu J, He ML, Wang L, Chen Y, Liu X, Dong Q, et al. Mir-26a inhibits cell growth and tumorigenesis of nasopharyngeal carcinoma through repression of ezh2. Cancer Res. 2011;71:225-33.

34. Yu L, Lu J, Zhang B, Liu X, Wang L, Li SY, et al. Mir-26a inhibits invasion and metastasis of nasopharyngeal cancer by targeting ezh2. Oncol Lett. 2013;5:1223-8.

35. Liu N, Tang LL, Sun Y, Cui RX, Wang HY, Huang BJ, et al. Mir-29c suppresses invasion and metastasis by targeting tiam1 in nasopharyngeal carcinoma. Cancer Lett. 2013;329:181-8.

36. Zhang JX, Qian D, Wang FW, Liao DZ, Wei JH, Tong ZT, et al. Microrna-29c enhances the sensitivities of human nasopharyngeal carcinoma to cisplatin-based chemotherapy and radiotherapy. Cancer Lett. 2013;329:91-8.

37. Alajez NM, Shi W, Hui AB, Bruce J, Lenarduzzi M, Ito E, et al. Enhancer of zeste homolog 2 (ezh2) is overexpressed in recurrent nasopharyngeal carcinoma and is regulated by mir-26a, mir-101, and mir-98. Cell Death Dis. 2010;21:64.

38. Xia H, Ng SS, Jiang S, Cheung WK, Sze J, Bian XW, et al. Mir-200amediated downregulation of zeb2 and ctnnb1 differentially inhibits nasopharyngeal carcinoma cell growth, migration and invasion. Biochem Biophys Res Commun. 2010;391:535-41.

39. Xia H, Cheung WK, Sze J, Lu G, Jiang S, Yao H, et al. Mir200a regulates epithelial-mesenchymal to stem-like transition via zeb2 and beta-catenin signaling. J Biol Chem. 2010;285: 36995-7004.

40. Wang L, Tian WD, Xu X, Nie B, Lu J, Liu X, et al. Epstein-Barr virus nuclear antigen 1 (ebna1) protein induction of epithelial-mesenchymal transition in nasopharyngeal carcinoma cells. Cancer. 2014;120:363-72.

41. Yang X, Ni W, Lei K. Mir-200b suppresses cell growth, migration and invasion by targeting notch1 in nasopharyngeal carcinoma. Cell Physiol Biochem. 2013;32:1288-98.

42. Bussing I, Slack FJ, Grosshans H. Let-7 microRNAs in development, stem cells and cancer. Trends Mol Med. 2008;14:400-9.

43. Wang YY, Ren T, Cai YY, He XY. Microrna let-7a inhibits the proliferation and invasion of nonsmall cell lung cancer cell line 95d by regulating K-Ras and hmga2 gene expression. Cancer Biother Radiopharm. 2013;28:131-7.

44. Wong TS, Man OY, Tsang CM, Tsao SW, Tsang RK, Chan JY, et al. MicroRNA let-7 suppresses nasopharyngeal carcinoma cells proliferation through downregulating c-myc expression. J Cancer Res Clin Oncol. 2011;137:415-22.

45. Cai K, Wan Y, Sun G, Shi L, Bao X, Wang Z. Let-7a inhibits proliferation and induces apoptosis by targeting ezh2 in nasopharyngeal carcinoma cells. Oncol Rep. 2012;28:2101-6. 
46. Ma L, Deng X, Wu M, Zhang G, Huang J. Down-regulation of miRNA-204 by lmp-1 enhances cdc42 activity and facilitates invasion of EBV-associated nasopharyngeal carcinoma cells. FEBS Lett. 2014;588:1562-70.

47. Deng M, Tang H, Zhou Y, Zhou M, Xiong W, Zheng Y, et al. Mir216b suppresses tumor growth and invasion by targeting KRAS in nasopharyngeal carcinoma. J Cell Sci. 2011;124:2997-3005.

48. Deng M, Liu JF, Gu YX, Zheng GP, ZM H. mir-216b suppresses cell proliferation and invasion by targeting PKCalpha in nasopharyngeal carcinoma cells. Zhonghua Zhong Liu Za Zhi. 2013;35:645-50.

49. Liu N, Jiang N, Guo R, Jiang W, He QM, Xu YF, et al. Mir-451 inhibits cell growth and invasion by targeting MIF and is associated with survival in nasopharyngeal carcinoma. Mol Cancer. 2013;12: 1476-4598.

50. Zhong W, He B, Quan T, Zhu C, Zhou S, Chen Y. Expression of mir143 in nasopharyngeal carcinoma cell lines and its effect on cell adhesion ability. Nan Fang Yi Ke Da Xue Xue Bao. 2013;33:582-5.

51. Zhong W, He B, Zhu C, Xiao L, Zhou S, Peng X. mir-143 inhibits migration of human nasopharyngeal carcinoma cells by negatively regulating gli3 gene. Nan Fang Yi Ke Da Xue Xue Bao. 2013;33: 1057-61.

52. Hui AB, Bruce JP, Alajez NM, Shi W, Yue S, Perez-Ordonez B, et al. Significance of dysregulated metadherin and microRNA-375 in head and neck cancer. Clin Cancer Res. 2011;17:7539-50.

53. Wu CD, Kuo YS, Wu HC, Lin CT. MicroRNA-1 induces apoptosis by targeting prothymosin alpha in nasopharyngeal carcinoma cells. J Biomed Sci. 2011;18:1423-0127.

54. Peng XH, Huang HR, Lu J, Liu X, Zhao FP, Zhang B, et al. Mir-124 suppresses tumor growth and metastasis by targeting foxq1 in nasopharyngeal carcinoma. Mol Cancer. 2014;13:186.

55. Liu X, Lv XB, Wang XP, Sang Y, Xu S, Hu K, et al. Mir-138 suppressed nasopharyngeal carcinoma growth and tumorigenesis by targeting the CCND1 oncogene. Cell Cycle. 2012;11:2495-506.

56. Zhen Y, Liu Z, Yang H, Yu X, Wu Q, Hua S, et al. Tumor suppressor pdcd4 modulates mir-184-mediated direct suppression of c-myc and bcl2 blocking cell growth and survival in nasopharyngeal carcinoma. Cell Death Dis. 2013;24:376.

57. Li T, Chen JX, Fu XP, Yang S, Zhang Z, Chen Kh H, et al. MicroRNA expression profiling of nasopharyngeal carcinoma. Oncol Rep. 2011;25:1353-63.

58. Luo Z, Dai Y, Zhang L, Jiang C, Li Z, Yang J, et al. Mir-18a promotes malignant progression by impairing microRNA biogenesis in nasopharyngeal carcinoma. Carcinogenesis. 2013;34:415-25.

59. Yu X, Zhen Y, Yang H, Wang H, Zhou Y, Wang E, et al. Loss of connective tissue growth factor as an unfavorable prognosis factor activates mir-18b by pi3k/akt/c-jun and cmyc and promotes cell growth in nasopharyngeal carcinoma. Cell Death Dis. 2013;16:153.

60. Zhang L, Deng T, Li X, Liu H, Zhou H, Ma J, et al. Microrna-141 is involved in a nasopharyngeal carcinoma-related genes network. Carcinogenesis. 2010;31:559-66.

61. Chen P, Guo X, Zhou H, Zhang W, Zeng Z, Liao Q, et al. Splunc1 regulates cell progression and apoptosis through the mir-141PTEN/p27 pathway, but is hindered by lmp1. PLoS ONE. 2013;8:5.

62. Du ZM, Hu LF, Wang HY, Yan LX, Zeng YX, Shao JY, et al. Upregulation of mir-155 in nasopharyngeal carcinoma is partly driven by $1 \mathrm{mp} 1$ and $1 \mathrm{mp} 2 \mathrm{a}$ and downregulates a negative prognostic marker jmjd1a. PLoS ONE. 2011;6:0019137.

63. Zhu X, Wang Y, Sun Y, Zheng J, Zhu D. Mir-155 up-regulation by lmp1 DNA contributes to increased nasopharyngeal carcinoma cell proliferation and migration. Eur Arch Otorhinolaryngol. 2014;271: 1939-45.

64. Deng M, Ye Q, Qin Z, Zheng Y, He W, Tang H, et al. Mir-214 promotes tumorigenesis by targeting lactotransferrin in nasopharyngeal carcinoma. Tumour Biol. 2013;34:1793-800.
65. Zhang ZC, Li YY, Wang HY, Fu S, Wang XP, Zeng MS, et al. Knockdown of mir-214 promotes apoptosis and inhibits cell proliferation in nasopharyngeal carcinoma. PLoS ONE. 2014;9.

66. Chen L, Tang Y, Wang J, Yan Z, Xu R. Mir-421 induces cell proliferation and apoptosis resistance in human nasopharyngeal carcinoma via downregulation of foxo4. Biochem Biophys Res Commun. 2013;435:745-50.

67. Li G, Wu Z, Peng Y, Liu X, Lu J, Wang L, et al. Microrna-10b induced by Epstein-Barr virus-encoded latent membrane protein-1 promotes the metastasis of human nasopharyngeal carcinoma cells. Cancer Lett. 2010;299:29-36.

68. Sun XJ, Liu H, Zhang P, Zhang XD, Jiang ZW, Jiang CC. Mir-10b promotes migration and invasion in nasopharyngeal carcinoma cells. Asian Pac J Cancer Prev. 2013;14:5533-7.

69. Li Y, Yan L, Zhang W, Wang H, Chen W, Hu N, et al. Mir-21 inhibitor suppresses proliferation and migration of nasopharyngeal carcinoma cells through down-regulation of $\mathrm{bcl} 2$ expression. Int $\mathrm{J}$ Clin Exp Pathol. 2014;7:3478-87.

70. Zhao Y, Chen X, Jing M, Du H, Zeng Y. Expression of miRNA-146a in nasopharyngeal carcinoma is upregulated by Epstein-Barr virus latent membrane protein 1. Oncol Rep. 2012;28:1237-42.

71. Lung RW, Wang X, Tong JH, Chau SL, Lau KM, Cheng SH, et al. A single nucleotide polymorphism in microrna-146a is associated with the risk for nasopharyngeal carcinoma. Mol Carcinog. 2013;52:18.

72. Yu BL, Peng XH, Zhao FP, Liu X, Lu J, Wang L, et al. Microrna-378 functions as an onco-miR in nasopharyngeal carcinoma by repressing TOB2 expression. Int J Oncol. 2014;44:1215-22.

73. Yi C, Wang Q, Wang L, Huang Y, Li L, Liu L, et al. Mir-663, a microRNA targeting $\mathrm{p} 21$ (wafl/cip1), promotes the proliferation and tumorigenesis of nasopharyngeal carcinoma. Oncogene. 2012;31: 4421-33.

74. Hurst DR, Edmonds MD, Welch DR. Metastamir: the field of metastasis-regulatory microRNA is spreading. Cancer Res. 2009;69:7495-8.

75. Leung TW, Tung SY, Sze WK, Wong FC, Yuen KK, Lui CM, et al. Treatment results of 1070 patients with nasopharyngeal carcinoma: an analysis of survival and failure patterns. Head Neck. 2005;27:555-65.

76. Lyu X, Fang W, Cai L, Zheng H, Ye Y, Zhang L, et al. Tgfbetar2 is a major target of mir-93 in nasopharyngeal carcinoma aggressiveness. Mol Cancer. 2014;13:1476-4598.

77. Wang HY, Li YY, Fu S, Wang XP, Huang MY, Zhang X, et al. MicroRNA-30a promotes invasiveness and metastasis in vitro and in vivo through epithelial-mesenchymal transition and results in poor survival of nasopharyngeal carcinoma patients. Exp Biol Med. 2014;239:891-8.

78. Zhang LY, Ho-Fun Lee V, Wong AM, Kwong DL, Zhu YH, Dong SS, et al. MicroRNA-144 promotes cell proliferation, migration and invasion in nasopharyngeal carcinoma through repression of PTEN. Carcinogenesis. 2013;34:454-63.

79. Luo Z, Zhang L, Li Z, Jiang C, Dai Y, Liu X, et al. Mir-149 promotes epithelial-mesenchymal transition and invasion in nasopharyngeal carcinoma cells. Zhong Nan Da Xue Xue Bao Yi Xue Ban. 2011;36: 604-9.

80. Chen SJ, Chen GH, Chen YH, Liu CY, Chang KP, Chang YS, et al. Characterization of Epstein-Barr virus miRNAome in nasopharyngeal carcinoma by deep sequencing. PLoS ONE. 2010;5:0012745.

81. Lo AK, Dawson CW, Jin DY, Lo KW. The pathological roles of BART miRNAs in nasopharyngeal carcinoma. J Pathol. 2012;227: 392-403.

82. Ye Y, Zhou Y, Zhang L, Chen Y, Lyu X, Cai L, et al. EBV-mir-BART 1 is involved in regulating metabolism-associated genes in nasopharyngeal carcinoma. Biochem Biophys Res Commun. 2013;436:1924.

83. Gourzones C, Ferrand FR, Amiel C, Verillaud B, Barat A, Guerin M, et al. Consistent high concentration of the viral microRNA BART17 
in plasma samples from nasopharyngeal carcinoma patients - evidence of non-exosomal transport. Virol J. 2013;10:10-119.

84. Lei T, Yuen KS, Xu R, Tsao SW, Chen H, Li M, et al. Targeting of dice1 tumor suppressor by Epstein-Barr virus-encoded mirBART3* microRNA in nasopharyngeal carcinoma. Int J Cancer. 2013;133:79-87.

85. Chan JY, Gao W, Ho WK, Wei WI, Wong TS. Overexpression of Epstein-Barr virus-encoded microRNA-BART7 in undifferentiated nasopharyngeal carcinoma. Anticancer Res. 2012;32:3201-10.

86. Jiang QP, Liu SY, He XF, Peng J, Xiong HZ, Xiong ZT, et al. Relationship between map3k5 and Epstein-Barr virus-encoded mirBART22 expression in nasopharyngeal carcinoma. Nan Fang Yi Ke Da Xue Xue Bao. 2011;31:1146-9.

87. Cai X, Wei W, Zhao S, Tang Y, He C, Wang C. Inhibition of the expression of VEGF gene in nasopharyngeal carcinoma cells by microRNA. Lin Chung Er Bi Yan Hou Tou Jing Wai Ke Za Zhi. 2010;24:703-7.

88. Gao W, Chan JY, Wong TS. Curcumin exerts inhibitory effects on undifferentiated nasopharyngeal carcinoma by inhibiting the expression of mir-125a-5p. Clin Sci. 2014;127:571-9.

89. Liu Y, Li Z, Wu L, Wang Z, Wang X, Yu Y, et al. Mirna-125a-5p: a regulator and predictor of gefitinib's effect on nasopharyngeal carcinoma. Cancer Cell Int. 2014;14:1475-2867.

90. Chen ZX, Sun AM, Chen Y, Liu Y, Zhan JF, Chen LH, et al. Effects of radiosensitivity and $\mathrm{X}$-ray dose on mir-7 expression in nasopharyngeal carcinoma. Nan Fang Yi Ke Da Xue Xue Bao. 2010;30:1810-2.

91. Qu C, Liang Z, Huang J, Zhao R, Su C, Wang S, et al. Mir-205 determines the radioresistance of human nasopharyngeal carcinoma by directly targeting PTEN. Cell Cycle. 2012;11:785-96.
92. Li G, Liu Y, Su Z, Ren S, Zhu G, Tian Y, et al. Microrna-324-3p regulates nasopharyngeal carcinoma radioresistance by directly targeting wnt2b. Eur J Cancer. 2013;49:2596-607.

93. Zheng XH, Cui C, Ruan HL, Xue WQ, Zhang SD, Hu YZ, et al. Plasma microRNA profiles of nasopharyngeal carcinoma patients reveal mir-548q and mir-483-5p as potential biomarkers. Chin J Cancer. 2014;26:10246.

94. Wang HY, Yan LX, Shao Q, Fu S, Zhang ZC, Ye W, et al. Profiling plasma microRNA in nasopharyngeal carcinoma with deep sequencing. Clin Chem. 2014;60:773-82.

95. Guo X, Liao Q, Chen P, Li X, Xiong W, Ma J, et al. The microRNAprocessing enzymes: Drosha and dicer can predict prognosis of nasopharyngeal carcinoma. J Cancer Res Clin Oncol. 2012;138:49 56.

96. Liu N, Cui RX, Sun Y, Guo R, Mao YP, Tang LL, et al. A fourmiRNA signature identified from genome-wide serum miRNA profiling predicts survival in patients with nasopharyngeal carcinoma. Int J Cancer. 2014;134:1359-68.

97. Zeng X, Xiang J, Wu M, Xiong W, Tang H, Deng M, et al. Circulating mir-17, mir-20a, mir-29c, and mir-223 combined as non-invasive biomarkers in nasopharyngeal carcinoma. PLoS ONE. 2012;7:8.

98. Liu N, Chen NY, Cui RX, Li WF, Li Y, Wei RR, et al. Prognostic value of a microRNA signature in nasopharyngeal carcinoma: a microRNA expression analysis. Lancet Oncol. 2012;13:633-41.

99. Wang LJ, Chou YF, Chen PR, Su B, Hsu YC, Chang CH, et al. Differential miRNA expression in repeated recurrence of nasopharyngeal carcinoma. Cancer Lett. 2014;344:188-94. 